\title{
Elektrische Kontakte zwischen identischen Halbleitern
}

\section{Doctoral Thesis}

Author(s):

Schade, Horst

Publication date:

1965

Permanent link:

https://doi.org/10.3929/ethz-a-000091395

Rights / license:

In Copyright - Non-Commercial Use Permitted 
Prom. Nr. 3555

\title{
Elektrische Kontakte zwischen identischen Halbleitern
}

\author{
Von der
}

\section{EIDGENOSSISCHEN TECHNISCHEN HOCHSCHULE IN ZURICH}

\author{
zur Erlangung \\ der Würde eines Doktors der \\ Naturwissenschaften \\ genehmigte \\ PROMOTIONSARBEIT \\ vorgelegt von \\ HORST SCHADE \\ dipl. Phys. ETH \\ deutscher Staatsangehöriger
}

Referent: Herr Prof. Dr. G. Busch

Korreferent: Herr Prof. Dr. H. Gränicher

Springer-Verlag

Berlin - Heidelberg · New York

1965 
Aus dem Laboratorium für Festkörperphysik

der Eidgenössischen Technischen Hochschule, Zürich

\title{
Elektrische Kontakte zwischen identischen Halbleitern
}

\author{
Von \\ H. SCHADE \\ Mit 19 Figuren
}

(Eingegangen am 25. September 1964)

Die Strom-Spannungs-Charakteristiken von Kontakten zwischen gereinigten und oxydierten Silizium-Oberflächen wurden im Temperaturbereich von $200^{\circ}$ bis $500^{\circ} \mathrm{K}$ gemessen.

Die experimentellen Ergebnisse sind erklärbar unter der Annahme einer durch Zwischenschichtzustände verursachten Potentialbarriere am Kontakt, deren Höhe vom jeweiligen Zustand der Oberflächen abhängt. Die quantitative Auswertung im Rahmen eines theoretischen Modells von Stratron hat ergeben, daß der Schottky-Effekt den Ladungsträgertransport durch den Kontakt wesentlich bestimmt.

Les caractéristiques courant-tension de contacts entre des surfaces de silicium nettoyées ou oxydées ont été mésurées entre $200^{\circ}$ et $500^{\circ} \mathrm{K}$.

Les résultats expérimentaux sont explicables, si l'on admet au contact une barrière de potentiel provenant des états d'interface et dont la grandeur dépend de l'état de la surface. Lianalyse quantitative utilisant le modèle théorique de Stratron a montré, que l'effet Schottky est prédominant dans le transport de charges au travers du contact.

The current-voltage characteristics of contacts between flash-cleaned and also between oxidized silicon surfaces have been determined in the temperature range from $200^{\circ}$ to $500^{\circ} \mathrm{K}$.

The results are interpreted under the assumption that a potential barrier caused by interface states exists within the contact region, and that the height of this barrier is given by the state of the contacting surfaces. Using the theory developed by STratToN, the charge carrier transport is shown to be predominantly determined by the Schottky effect.

\section{A. Einleitung}

Elektrische Kontakte zwischen identischen Halbleitern haben im allgemeinen eine nicht-ohmsche Strom-Spannungs-Charakteristik: der Kontaktwiderstand sinkt mit steigender Spannung.

Es sind viele Untersuchungen über Halbleiter-Halbleiter-Kontakte bekannt und in einer ausführlichen Arbeit von ZüCKLER [1], [2] zusammengefaßt. Meistens wurde der Kontaktwiderstand zwischen Siliziumkarbid-Kristallen studiert, dessen Verhalten im Hinblick auf elektrotechnische Anwendungen von Bedeutung ist. Als Erklärung für die starke Spannungsabhängigkeit des Kontaktwiderstandes wurden folgende Phänomene diskutiert:

Tunneleffekt durch eine isolierende Zwischenschicht am Kontakt (BraUN und Busch [3], Dilworth [4], Mitchelu und Sillars [5], R. Holm [6], E. Holm [7], Heywang [8], Strässler [9]), 